\title{
ARTICLE
}

Clinical Study

\section{Neoadjuvant PF-05280014 (a potential trastuzumab biosimilar) versus trastuzumab for operable HER2+ breast cancer}

\author{
Philip E. Lammers ${ }^{1}$, Magdolna Dank ${ }^{2}$, Riccardo Masetti ${ }^{3}$, Richat Abbas ${ }^{4}$, Fiona Hilton ${ }^{5}$, Jennifer Coppola ${ }^{6}$ and Ira Jacobs $^{6}$
}

BACKGROUND: This randomised, double-blind study compared pharmacokinetics, efficacy, safety and immunogenicity of PF-05280014 (potential trastuzumab biosimilar) and trastuzumab reference product (Herceptin) sourced from the European Union (trastuzumab-EU) as neoadjuvant treatment for operable human epidermal growth factor receptor 2 (HER2)-positive breast cancer. METHODS: Patients $(N=226)$, stratified by primary tumour size and hormone receptor status, were randomised 1:1 to PF-05280014 or trastuzumab-EU ( $8 \mathrm{mg} / \mathrm{kg}$ loading dose; $6 \mathrm{mg} / \mathrm{kg}$ thereafter), each with docetaxel and carboplatin, every 3 weeks for six treatment cycles. Primary endpoint was percentage of patients with trough plasma concentration $\left(C_{\text {trough }}\right)>20 \mu \mathrm{g} / \mathrm{ml}$ at Cycle 5 (Cycle 6 predose). Efficacy endpoints included pathological complete response and objective response rate. Non-inferiority of PF-05280014 to trastuzumab-EU was declared if the lower limit of the $95 \%$ confidence interval for the stratified difference between groups in the percentage of patients with Cycle $5 C_{\text {trough }}>20 \mu \mathrm{g} / \mathrm{ml}$ was above the prespecified non-inferiority margin of $-12.5 \%$. RESULTS: For PF-05280014 vs trastuzumab-EU patients, respectively, $92.1 \%$ vs $93.3 \%$ had Cycle $5 C_{\text {trough }}>20 \mu \mathrm{g} / \mathrm{ml}$; the lower limit of the $95 \%$ confidence interval $(-8.02 \%, 6.49 \%)$ for the stratified difference between groups was above the non-inferiority margin $(-12.5 \%)$. Pathological complete response $(47.0 \%$ vs $50.0 \%)$ and central radiology review-assessed objective response $(88.1 \%$ vs $82.0 \%$ ) rates were comparable. Incidence of all-causality, grade 3-4 treatment-emergent adverse events was $38.1 \%$ vs $45.5 \%$; antidrug antibody rates were $0 \%$ vs $0.89 \%$.

CONCLUSIONS: PF-05280014 demonstrated non-inferior pharmacokinetics and comparable efficacy, safety and immunogenicity to trastuzumab-EU in patients with operable HER2-positive breast cancer receiving neoadjuvant chemotherapy.

British Journal of Cancer (2018) 119:266-273; https://doi.org/10.1038/s41416-018-0147-1

\section{INTRODUCTION}

Trastuzumab is a recombinant humanised immunoglobulin G1 monoclonal antibody that targets human epidermal growth factor receptor 2 (HER2) and is approved for the treatment of HER2overexpressing breast and gastric cancers. ${ }^{1,2}$ HER2 overexpression occurs in $15-20 \%$ of invasive breast cancers; it is associated with more-aggressive biological behaviour and, in the absence of treatment with HER2-targeted therapy, worse clinical outcomes. ${ }^{3-6}$ The addition of trastuzumab to adjuvant chemotherapy for HER2positive (HER2+) early breast cancer or to chemotherapy for HER2 + metastatic disease reduces the risk for recurrence or disease progression and prolongs survival as compared with chemotherapy alone. ${ }^{7-10}$ When added to neoadjuvant chemotherapy, trastuzumab improves rates of event-free survival and pathological complete response (pCR) over chemotherapy alone. ${ }^{11,12}$

Despite the clinical benefits associated with HER2-directed therapy, physicians worldwide often encounter barriers to prescribing trastuzumab, leading to less than optimal treatment of patients with HER2+ breast cancer. ${ }^{13,14}$ The availability of biosimilars may expand access to biologic therapies such as trastuzumab and provide patients with additional safe and efficacious treatment options. Biosimilars are biologic drugs that are highly similar to a licensed (i.e., originator or reference) biologic product. ${ }^{15-17}$ To receive regulatory approval, a proposed biosimilar product must show no clinically meaningful differences in safety, purity or potency compared with the originator biologic, based on the totality of the evidence obtained from comparative assessments of the two products. ${ }^{15-17}$ Regulatory agencies recommend a stepwise approach to generating these data that begins with comprehensive analytical (i.e., structural and functional) characterisation followed by nonclinical testing and culminates with a comparative clinical study (or studies) to confirm similarity between the proposed biosimilar and originator product in pharmacokinetics, efficacy, safety and immunogenicity. ${ }^{15-17}$

PF-05280014 is under development as a potential biosimilar of trastuzumab (Herceptin). Comparative non-clinical assessments of PF-05280014 and trastuzumab reference products marketed in the European Union (trastuzumab-EU; Herceptin, Roche Registration $\mathrm{GmbH}$, Grenzach-Wyhlen, Germany) and United States

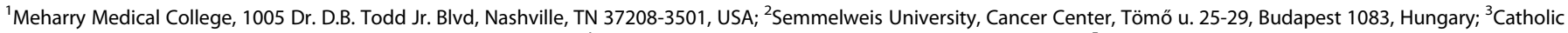

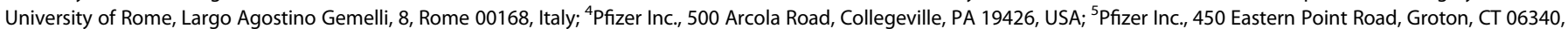
USA and ${ }^{6}$ Pfizer Inc., 235 and 219 East 42nd Street, New York, NY 10017-5755, USA

Correspondence: Ira Jacobs (ira.jacobs@pfizer.com)

Received: 31 August 2017 Revised: 26 April 2018 Accepted: 25 May 2018

Published online: 13 July 2018 
(trastuzumab-US; Herceptin, South San Francisco, CA) demonstrated PF-05280014 has the same primary amino-acid sequence as the licensed trastuzumab, with similar in vitro functional properties and in vivo pharmacokinetics, antidrug antibody (ADA) responses and tolerability. ${ }^{18}$ Furthermore, two single-dose comparability studies conducted in healthy male volunteers demonstrated PF-05280014, trastuzumab-EU and trastuzumab-US have similar pharmacokinetics, safety and immunogenicity profiles. ${ }^{19,20}$ An additional, distinct comparative safety and efficacy study (NCT01989676) is evaluating PF-05280014 versus trastuzumab-EU, each administered in combination with paclitaxel, as first-line treatment for patients with HER2+ metastatic breast cancer. ${ }^{21}$

We report the results of a comparative clinical trial conducted to compare pharmacokinetics, efficacy, safety and immunogenicity of PF-05280014 versus trastuzumab-EU, each administered in combination with docetaxel and carboplatin, as neoadjuvant treatment for patients with operable HER2 + breast cancer. ${ }^{22}$ It was hypothesised that PF-05280014 was non-inferior to trastuzumab-EU, based on pharmacokinetics data.

\section{METHODS}

Study population

Eligible patients were women aged 18 years or older with histologically confirmed invasive breast cancer that exhibited HER2 gene amplification by fluorescent in situ hybridisation, chromogenic in situ hybridisation or dual in situ hybridisation, as defined by the manufacturer's kit instruction; or HER2 overexpression by immunohistochemistry (IHC) categorised as $\mathrm{IHC} 3+$; or HER2 overexpression by IHC categorised as $\mathrm{IHC} 2+$ with fluorescent, chromogenic, or dual in situ hybridisation confirmation. HER2 + tumour status was determined by the site at the time of diagnosis, using either a sponsor-approved assay or two different analytical test methods that were not considered sponsor-approved but both demonstrated unequivocal (i.e., IHC3+) results, and confirmed retrospectively by the sponsorprovided central laboratory. If tumour HER2 status could not be determined via local testing, it was evaluated by central laboratory assessment.

Patients with measurable disease (longest diameter $\geq 2.0 \mathrm{~cm}$ ) in the breast after diagnostic biopsy and known hormone (oestrogen and progesterone) receptor status at study entry were included. For patients with unknown hormone receptor status, oestrogen and progesterone receptor status testing was performed at screening, via local or central laboratory assessment. Oestrogen and progesterone receptor positivity was determined by local site guidelines based on accepted standards. Baseline tumour assessments were performed within 6 weeks prior to randomisation and included computed tomography (CT), or magnetic resonance imaging of the chest if a CT scan could not be performed, and bilateral mammography or ultrasound of the breast. Patients were planned to undergo definitive surgical resection of breast tumour (i.e., lumpectomy or mastectomy with sentinel node biopsy or axillary lymph node dissection) and neoadjuvant chemotherapy. Other inclusion criteria included: Eastern Cooperative Oncology Group performance status $0-1$; left ventricular ejection fraction (LVEF) $\geq 55 \%$ as measured by two-dimensional echocardiogram $(\mathrm{ECHO})$ or multi-gated acquisition scan (MUGA); and normal laboratory values.

Key exclusion criteria included bilateral breast cancer; inflammatory breast cancer; presence of known distant metastases, as determined by the investigator; prior chemotherapy, endocrine therapy, biologic therapy, radiation or surgery, except diagnostic biopsy for primary breast cancer; other concomitant active malignancy or history of malignancy in the past 5 years, except treated basal cell carcinoma of the skin or carcinoma in situ of the cervix; history of documented or current congestive heart failure; current high-risk uncontrolled arrhythmias; angina pectoris requiring treatment; clinically significant valvular disease; evidence of transmural infarction on electrocardiogram; or poorly controlled hypertension.

Study design, procedures and treatments

This was an international, double-blind, randomised clinical trial initiated at 67 sites in 10 countries across Europe (EudraCT registration number 2013-004679-11) and in the United States (ClinicalTrials.gov identifier NCT02187744). ${ }^{22,23}$

Patients who satisfied the eligibility criteria were stratified by primary tumour size $(<5 \mathrm{~cm}$ vs $\geq 5 \mathrm{~cm})$ and hormone receptor status (positive vs negative) and randomised $1: 1$ to receive PF-05280014 or trastuzumab-EU (Herceptin), each given in combination with docetaxel and carboplatin (Fig. 1a). On Day 1 Cycle 1, patients received a loading dose $(8 \mathrm{mg} / \mathrm{kg}$, over $90-\mathrm{min}$ intravenous (IV) infusion) of PF-05280014 or trastuzumab-EU followed by docetaxel $\left(75 \mathrm{mg} / \mathrm{m}^{2} ; 60-\mathrm{min}\right.$ IV infusion) and carboplatin (target area under the curve: 6 ; $\geq 15$-min IV infusion). Subsequent infusions of PF-05280014 or trastuzumab-EU $(6 \mathrm{mg} /$ $\mathrm{kg}$, over 30 to $90 \mathrm{~min}$ ), docetaxel and carboplatin were administered every 3 weeks for six treatment cycles. Neoadjuvant therapy with trastuzumab in combination with docetaxel and carboplatin has demonstrated efficacy and safety in patients with HER2+ early breast cancer. ${ }^{24}$ Furthermore, the trastuzumab treatment regimen used in this study is consistent with trastuzumab-EU product labelling and is in line with the National Comprehensive Cancer Network recommendations on the preferred preoperative/adjuvant therapy regimens for HER2 + breast cancer. ${ }^{2,25}$ Patients who experienced toxicity attributed to PF05280014 or trastuzumab-EU were required to temporarily or permanently discontinue trastuzumab treatment; dose reductions were not permitted. Granulocyte colony-stimulating factor was used for prophylactic or therapeutic management of haematologic toxicities attributed to docetaxel. Reductions in the docetaxel dose or discontinuation of treatment with docetaxel were permitted.

Patients had a follow-up assessment, i.e., end of treatment (EOT) visit, 28 ( \pm 7$)$ days after final study drug administration and underwent definitive surgical resection of their primary breast tumour within 3-7 weeks following the EOT visit. Systemic therapy given after resection was not mandated by the protocol and PF-05280014 or trastuzumab-EU was not provided for treatment in the adjuvant setting. Patients were not followed for clinical endpoints after resection.

Objectives and endpoints

The primary objective was to determine whether PF-05280014 is non-inferior to trastuzumab-EU, based on pharmacokinetics data. The mechanism of action of trastuzumab is mediated through its binding to the target receptor HER2, and full receptor saturation is expected to drive efficacy of trastuzumab. ${ }^{1,2,26}$ Accordingly, similar trough plasma concentrations $\left(C_{\text {trough }}\right)$ for PF-05280014 and trastuzumab-EU would support similar efficacy between treatments. Furthermore, $C_{\text {trough }}>20 \mu \mathrm{g} / \mathrm{ml}$ is a therapeutic target threshold concentration for trastuzumab and has been used to demonstrate noninferiority of subcutaneous trastuzumab to intravenous trastuzumab in the neoadjuvant setting. ${ }^{26}$ Therefore, non-inferiority was assessed by comparing the percentage of patients with Cycle $5 C_{\text {trough }}$ (Cycle 6 predose) $>20 \mu \mathrm{g} / \mathrm{ml}$ (primary endpoint) in the PF-05280014 group with that in the trastuzumab-EU group. This time point (Cycle 5 $C_{\text {trough }}$ ) was selected because it would be expected to reflect steady state drug concentration based on population predicted pharmacokinetic exposure values for the approved 3-weekly dosing regimen used in this study. ${ }^{2}$

Secondary objectives were to evaluate the efficacy, safety, immunogenicity and other pharmacokinetic measures of PF-05280014 and trastuzumab-EU. Efficacy was measured by the percentage of patients with $\mathrm{pCR}$, defined as the absence of 

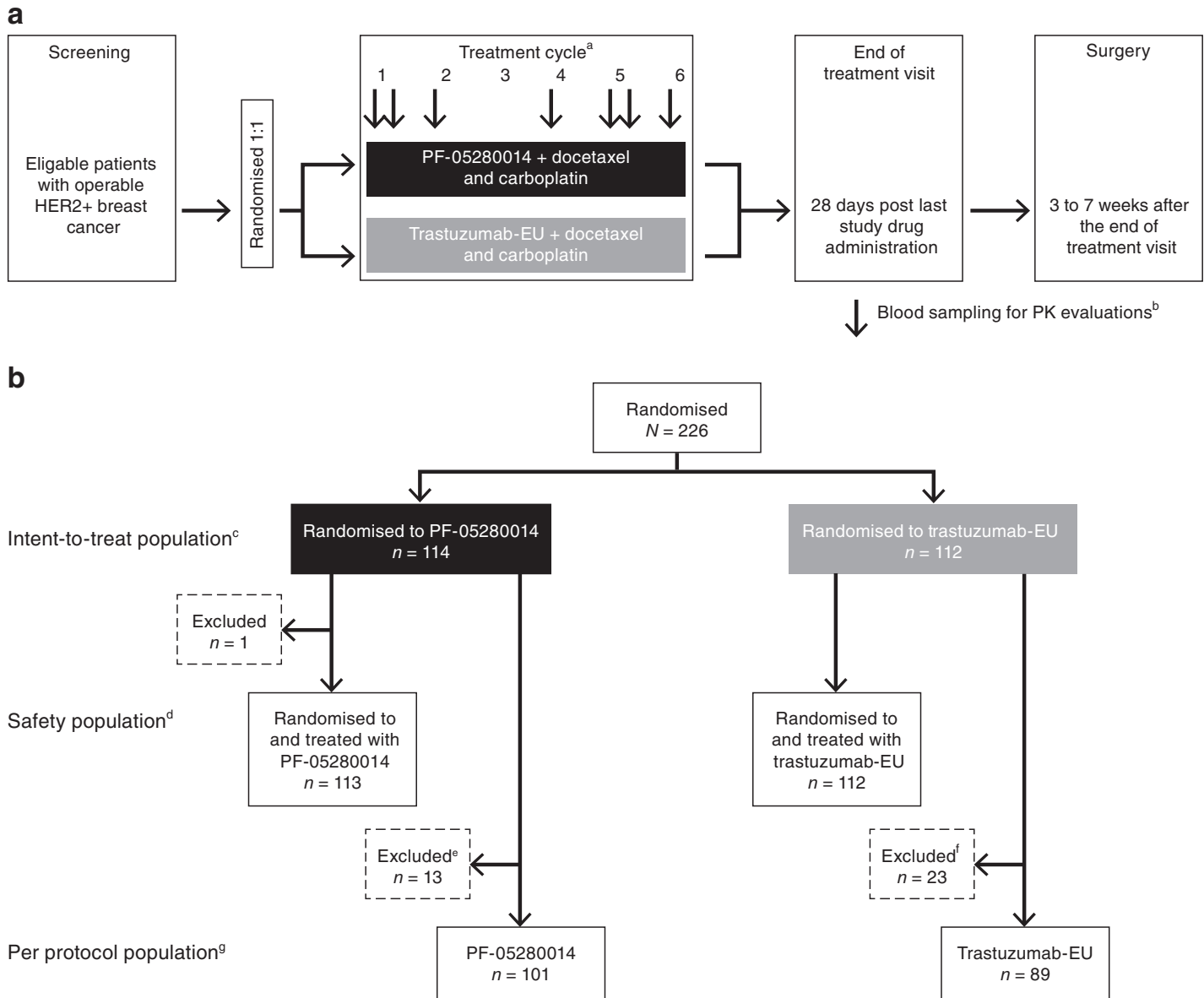

Fig. 1 Overall study design and disposition of patients. a Study design and $\mathbf{b}$ patient disposition. ${ }^{\mathrm{a}}$ On Day 1 Cycle 1 , patients received a loading dose $(8 \mathrm{mg} / \mathrm{kg})$ of PF-05280014 or trastuzumab-EU infused over $90 \mathrm{~min}$ followed by docetaxel $\left(75 \mathrm{mg} / \mathrm{m}^{2} ; 60-\mathrm{min}\right.$ intravenous infusion) and carboplatin (target AUC: $6 ; \geq 15$-min intravenous infusion). Subsequent infusions of PF-05280014 or trastuzumab-EU (6 mg/kg, over 30 to $90 \mathrm{~min}$ ), docetaxel and carboplatin were administered every 3 weeks for a total of six treatment cycles. ${ }^{\mathrm{b}}$ Blood samples were collected predose ( $-2.5 \mathrm{~h}$ to -5 min prior to infusion) on Day 1 of Cycles $1,2,4,5$ and 6 , and at 1-h post dose on Day 1 of Cycles 1 and 5 for determination of PF-05280014 or trastuzumab-EU serum concentrations. ${ }^{\mathrm{C}}$ The intent-to-treat population consisted of all patients randomised to PF-05280014 or trastuzumab-EU. ${ }^{\mathrm{d}}$ The safety population comprised all patients who received at least one dose of study drug. ${ }^{e}$ Patients in the PF-05280014 group were excluded from the per protocol population for the following reasons: Cycle 5 trough sample taken outside protocol-specified window $(n=6,46.2 \%)$, fewer than six cycles of trastuzumab $(n=5,38.5 \%)$, no Cycle 5 trough pharmacokinetic sample $(n=2,15.4 \%)$, and trastuzumab treatment delay $>1$ week $(n=1,7.7 \%)$. A patient may have met multiple criteria for exclusion and may have been counted more than once. fPatients in the trastuzumab-EU group were excluded from the per protocol population for the following reasons: Cycle 5 trough sample taken outside protocol-specified window $(n=13,56.5 \%)$, fewer than six cycles of trastuzumab $(n=5,21.7 \%)$, trastuzumab treatment delay $>1$ week $(n=3,13.0 \%)$, Cycle 5 trough sample taken post dose $(n=1,4.3 \%)$, no lesion $>2 \mathrm{~cm}$ in breast $(n=1$, 4.3\%), and missing HER2 sample $(n=1,4.3 \%)$. A patient may have met multiple criteria for exclusion and may have been counted more than once. ${ }^{9}$ The per protocol population consisted of all randomised patients who received six cycles of PF-05280014 or trastuzumab-EU and had no temporary delays in treatment lasting $>1$ week or other significant protocol deviations. AUC $=$ area under the curve; HER2 $=$ human epidermal growth factor receptor 2; trastuzumab-EU = licensed trastuzumab sourced from the European Union

invasive neoplastic cells in the breast and lymph nodes following surgery after treatment completion (remaining ductal carcinoma in situ was accepted), ${ }^{26}$ and objective response rate (ORR), defined as the percentage of patients who had complete or partial response at Cycle 6/EOT. Safety was characterised by the type, incidence, severity, timing, seriousness and relatedness of adverse events (AEs) to study drug, including cardiotoxicity, signs and symptoms of anaphylaxis, infusion-related reactions and laboratory abnormalities. Immunogenicity was measured by the incidence of ADAs, including neutralising antibodies (NAbs). Pharmacokinetics was further assessed by measuring $C_{\text {trough }}$ at Cycles 1 (Cycle 2 predose), 3 (Cycle 4 predose) and 4 (Cycle 5 predose), as well as $C_{\max }$ (maximum drug concentration, $1 \mathrm{~h}$ post dose) at Cycles 1 and 5.
Assessments

Blood samples for determining PF-05280014 or trastuzumab-EU serum concentrations were collected predose $(-2.5 \mathrm{~h}$ to $-5 \mathrm{~min}$ prior to infusion) on Day 1 of Cycles 1, 2, 4, 5 and 6, and at 1-h post dose on Day 1 of Cycles 1 and 5. Samples were analysed using a validated enzyme-linked immunosorbent assay with a lower limit of quantification of $0.500 \mu \mathrm{g} / \mathrm{ml}$. Pathological response status was determined by an investigator-designated qualified pathologist. Tumour assessments were performed at screening, end of Cycle 3, EOT and as clinically needed. Objective response status was determined by a central radiology laboratory and by the investigators using Response Evaluation Criteria in Solid Tumours, version 1.1. Central radiology assessments were used to calculate ORR at Cycle 6/EOT. 
AEs were monitored continuously from the time the patient received at least one dose of study drug through the last patient visit. The severity of AEs was graded in accordance with the National Cancer Institute Common Terminology Criteria for Adverse Events, version 4.03. Cardiac monitoring (MUGA scan or ECHO) was performed at screening, end of Cycle 3, EOT visit and as clinically indicated. Serum samples for detecting ADAs and NAbs were collected on Day 1 (predose) of Cycles 1, 2, 4 and 6 (Fig. 1a), and during the EOT visit. Samples were tested for ADAs following a tiered approach of screening, confirmation and titre. Samples were first analysed using a validated electrochemiluminescent immunoassay specific to PF-05280014 or trastuzumab-EU. ADA-positive samples were then analysed for cross-reactivity using the alternate assay. Confirmed ADA-positive samples were tested for NAb using a validated electrochemiluminescent immunoassay specific to the monoclonal antibody administered; NAb-positive samples were then analysed for cross-reactivity using the alternate assay.

Statistical methods

This study tested the hypothesis that the percentage of patients exhibiting Cycle $5 C_{\text {trough }}$ (Cycle 6 predose) $>20 \mu \mathrm{g} / \mathrm{ml}$ in the PF-05280014 group was non-inferior to that in the trastuzumabEU group, using a margin of $-12.5 \%$. This margin was selected based on clinical considerations rather than statistical considerations (e.g., a meta-analysis of previous data) as there is little previous evidence in the neoadjuvant setting outside of the HannaH trial, which evaluated non-inferiority of subcutaneous trastuzumab to intravenous trastuzumab based on $\mathrm{pCR}$ rate and using a margin of $-12.5 \% .^{26} \mathrm{~A}$ minimum of 188 patients ( $n=94 /$ arm) was required to provide $85 \%$ power to test for non-inferiority in the primary analysis. Considering a possible $15 \%$ attrition rate, a total sample size of $\sim 220$ patients $(n=110 / \mathrm{arm})$ was planned to ensure the required minimum number of patients for the non-inferiority analysis.

Differences between groups in the percentage of patients with Cycle $5 C_{\text {trough }}$ (Cycle 6 predose) $>20 \mu \mathrm{g} / \mathrm{ml}$, the percentage of patients who had $\mathrm{pCR}$ and the ORR were estimated, along with corresponding $95 \%$ confidence intervals, using the normal approximation to the binomial distribution and adjusting for randomisation strata. If the lower limit of the $\mathrm{Cl}$ for the primary endpoint was above $-12.5 \%$, the null hypothesis was rejected and PF-05280014 was considered non-inferior to trastuzumab-EU. Cycle $5 C_{\text {trough }}$ (Cycle 6 predose) values were log-transformed and the geometric ratio (PF-05280014 to trastuzumab-EU) of the means and corresponding $95 \% \mathrm{Cl}$ were also estimated (secondary pharmacokinetics endpoint).

Pharmacokinetics and efficacy analyses were performed in the per protocol population, defined as all randomised patients who received six cycles of PF-05280014 or trastuzumab-EU and had no temporary delays in treatment that lasted $>1$ week and no other significant protocol deviations. Sensitivity analyses of the primary and efficacy endpoints were performed in the intent-to-treat population (i.e., all randomised patients). Safety analyses, including $A E, A D A$ and $N A b$ analyses, were performed using the safety population (i.e., all patients who received at least one dose of study drug).

\section{RESULTS}

Patient disposition, demographics and baseline characteristics A total of 226 patients were randomised to PF-05280014 $(n=114)$ or trastuzumab-EU $(n=112)$ and were included in the intent-totreat population. Of these, one patient was randomised to but did not receive treatment with PF-05280014; the remaining 225 patients received study treatment as assigned and were included in the safety population (Fig. 1b). Of the 226 randomised patients, 190 (PF-05280014, $n=101$; trastuzumab-EU, $n=89$ ) met the
Table 1. Baseline demographics (intent-to-treat population) ${ }^{\mathrm{a}}$

\begin{tabular}{|c|c|c|c|}
\hline & $\begin{array}{l}\text { PF-05280014 } \\
(n=114)\end{array}$ & $\begin{array}{l}\text { Trastuzumab-EU } \\
(n=112)\end{array}$ & $\begin{array}{l}\text { Total } \\
(N=226)\end{array}$ \\
\hline Age, mean $( \pm S D)$, years & $54.0(11.9)$ & $51.2(12.7)$ & $52.6(12.3$ \\
\hline \multicolumn{4}{|l|}{ Race, $n$ (\%) } \\
\hline White & $112(98.2)$ & $109(97.3)$ & $221(97.8)$ \\
\hline Black & $1(0.9)$ & 0 & $1(0.4)$ \\
\hline Asian & $1(0.9)$ & $3(2.7)$ & $4(1.8)$ \\
\hline \multicolumn{4}{|l|}{ Ethnicity, $n$ (\%) } \\
\hline Hispanic/Latino & 0 & $1(0.9)$ & $1(0.4)$ \\
\hline Not Hispanic/Latino & $114(100.0)$ & $111(99.1)$ & $225(99.6)$ \\
\hline $\begin{array}{l}\text { Mean body mass } \\
\text { index }( \pm S D), \mathrm{kg} / \mathrm{m}^{2}\end{array}$ & $28.2(5.9)$ & $27.7(6.2)$ & $27.9(6.1)$ \\
\hline \multicolumn{4}{|c|}{ Primary tumour size, $n(\%)$} \\
\hline$<5 \mathrm{~cm}$ & $89(78.1)$ & $89(79.5)$ & $178(78.8)$ \\
\hline$\geq 5 \mathrm{~cm}$ & $25(21.9)$ & $23(20.5)$ & $48(21.2)$ \\
\hline \multicolumn{4}{|c|}{ Oestrogen receptor status, $n$ (\%) } \\
\hline Positive & $58(50.9)$ & $54(48.2)$ & $112(49.6)$ \\
\hline Negative & $56(49.1)$ & $58(51.8)$ & $114(50.4)$ \\
\hline \multicolumn{4}{|c|}{ Progesterone receptor status, $n(\%)$} \\
\hline Positive & $41(36.0)$ & $40(35.7)$ & $81(35.8)$ \\
\hline Negative & $73(64.0)$ & $72(64.3)$ & $145(64.2)$ \\
\hline
\end{tabular}

requirements for the per protocol population, and 36 (PF-05280014, $n=13$; trastuzumab-EU, $n=23$ ) were excluded for one or more of the following reasons, which were determined prior to breaking the blind (Fig. 1b): Cycle 5 trough sample taken outside the protocol-specified window, Cycle 5 trough sample taken post dose, fewer than six cycles of trastuzumab treatment, no Cycle 5 trough pharmacokinetics sample, no lesion $>2 \mathrm{~cm}$ in breast, missing HER2 sample and/or trastuzumab treatment delay $>1$ week. Patient demographics and other baseline characteristics for the intent-to-treat population were comparable between treatment groups (Table 1).

Pharmacokinetic analyses

In an analysis of the primary endpoint using the per protocol population, 93 (92.1\%) patients treated with PF-05280014 and 83 (93.3\%) patients treated with trastuzumab-EU exhibited Cycle 5 $C_{\text {trough }}$ (Cycle 6 predose) $>20 \mu \mathrm{g} / \mathrm{ml}$ (Table 2). The stratified estimated difference between PF-05280014 and trastuzumab-EU was $-0.76 \%$, and the lower limit of the $95 \% \mathrm{Cl}(-8.02 \%, 6.49 \%)$ was above the non-inferiority margin of $-12.5 \%$. In an analysis of secondary pharmacokinetics endpoints using the per protocol population, the geometric mean $C_{\text {trough }}$ at Cycle 5 was 34.59 and $34.56 \mu \mathrm{g} / \mathrm{ml}$ for PF-05280014 and trastuzumab-EU, respectively; the PF-05280014 to trastuzumab-EU ratio was $100.06 \%$ (95\% Cl: $81.5 \%, 122.9 \%)$. In addition, at each study cycle, trastuzumab serum concentrations appeared comparable between PF-05280014 and trastuzumab-EU (Supplementary Tables S1 and S2).

\section{Efficacy analyses}

Pathologic response and overall tumour response assessments for the per protocol population are summarised in Table 3. Of the patients in the PF-05280014 $(n=100)$ and trastuzumab-EU $(n=86)$ groups who had surgery, $47.0 \%(95 \% \mathrm{Cl}: 36.9 \%, 57.2 \%)$ 
Table 2. Primary pharmacokinetics analysis of patients reporting Cycle $5 C_{\text {trough }}{ }^{a}>20 \mu \mathrm{g} / \mathrm{ml}$ (per protocol population)

\begin{tabular}{lcc}
\hline & PF-05280014 $(n=101)$ & Trastuzumab-EU ( $n=89)$ \\
\hline Patients with Cycle $5 C_{\text {trough }}{ }^{\mathrm{a}}>20 \mu \mathrm{g} / \mathrm{ml}, \%(95 \% \mathrm{Cl})$ & $92.1(85.0,96.5)$ & $93.3(85.9,97.5)$ \\
Stratified difference between PF-05280014 and trastuzumab-EU ${ }^{\mathrm{b}}$ & -0.76 \\
Standard error for the difference & 3.70 \\
$95 \% \mathrm{Cl}$ (stratified) for the difference & $-8.02,6.49$ \\
\hline${ }^{\mathrm{a}} \mathrm{Cycle} 6$ predose. ${ }^{\mathrm{b}}$ Stratified analysis was based on the normal approximation to the binomial distribution, adjusting for randomisation strata (primary tumour \\
size $<5 \mathrm{~cm}$ vs $\geq 5 \mathrm{~cm} ;$ oestrogen receptor-positive vs oestrogen receptor-negative; and progesterone receptor-positive vs progesterone receptor-negative). \\
$\mathrm{Cl}=$ confidence interval; $C_{\text {trough }}=$ trough plasma concentration; trastuzumab-EU = licensed trastuzumab sourced from the European Union
\end{tabular}

and $50.0 \%$ (95\% Cl: $39.0 \%, 61.0 \%)$, respectively, had a $\mathrm{pCR}$; the stratified estimated difference between groups was $-2.81 \%$ (95\% Cl: $-16.58 \%, 10.96 \%)$. The ORR at Cycle 6/EOT was $88.1 \%$ (95\% Cl: $80.2 \%, 93.7 \%)$ for PF-05280014 and $82.0 \%$ (95\% Cl: $72.5 \%$, 89.4\%) for trastuzumab-EU, based on central radiology assessments; the stratified estimated difference between groups was $5.96 \%$ (95\% Cl: $-4.01 \%, 15.94 \%)$.

\section{Safety}

Among patients included in the safety population (PF-05280014, $n=113$; trastuzumab-EU, $n=112), 109 \quad(96.5 \%)$ in the PF-05280014 group and 109 (97.3\%) in the trastuzumab-EU group received six cycles of trastuzumab. Six patients each in the PF-05280014 (5.3\%) and trastuzumab-EU (5.4\%) groups had a delay in trastuzumab treatment. Eight (7.1\%) patients administered PF-05280014 and 7 (6.3\%) who received trastuzumab-EU had an interrupted trastuzumab infusion.

The majority of all patients experienced at least one treatmentemergent AE (TEAE) owing to any cause, with a total of 569 events reported by 109 (96.5\%) patients in the PF-05280014 group and 511 events reported by 106 (94.6\%) patients in the trastuzumabEU group (Table 4). The incidence of TEAEs and serious TEAEs was comparable between groups. The TEAEs most frequently reported by patients $(n(\%))$ in the PF-05280014 and trastuzumab-EU groups, respectively, were alopecia (72 (63.7\%) and $69(61.6 \%))$, anaemia (56 (49.6\%) and $51(45.5 \%))$ and neutropaenia (38 (33.6\%) and $41(36.6 \%))$. Grade 3-4 TEAEs were reported in $43(38.1 \%)$ and $51(45.5 \%)$ patients in the PF-05280014 and trastuzumab-EU groups, respectively. Seven (6.2\%) patients treated with PF-05280014 experienced seven serious AEs (SAEs; febrile neutropaenia, neutropaenia, pancytopenia, proctitis, devicerelated sepsis, injection-site abscess and increased blood creatinine); six (5.4\%) patients treated with trastuzumab-EU experienced 10 SAEs (anaemia, febrile neutropaenia $(n=2)$, neutropaenia (two SAEs, $n=1$ ), gastrointestinal infection, tooth infection, hip fracture, dehydration and hypokalaemia).

No TEAEs indicative of infusion-related reactions were reported in the PF-05280014 group; two $(1.8 \%)$ patients in the trastuzumab-EU group experienced non-serious events of pyrexia and tachypnea. Four (3.5\%) and three (2.7\%) patients in the PF-05280014 and trastuzumab-EU groups, respectively, permanently discontinued from any treatment due to AEs during the treatment period. One patient in the PF-05280014 group died due to a treatment-related SAE of pancytopenia; no other patients died during the study. No TEAEs of congestive heart failure or clinically significant LVEF abnormalities were reported by patients in either treatment group. There were no notable differences between the treatment groups in mean LVEF results (Supplementary Table S3). One $(0.88 \%)$ and $10(8.93 \%)$ patients in the PF-05280014 and trastuzumab-EU groups, respectively, had a decline in LVEF of $\geq 10 \%$ from baseline. However, no patient in either group had LVEF $<53 \%$ at any time point measured. Furthermore, no individual abnormalities or shifts in laboratory values were considered clinically relevant.
Immunogenicity

No patients in the PF-05280014 group and one $(0.89 \%)$ patient in the trastuzumab-EU group had positive ADA titres (Table 5). The single positive ADA titre of 2.39 was recorded at predose Cycle 1 . This patient also recorded a cross-reactivity ADA assay titre of 2.58, but was negative in all subsequent ADA tests and also tested negative for NAbs.

\section{DISCUSSION}

The primary objective of this comparative clinical trial in patients with operable HER2 + breast cancer was to determine whether neoadjuvant treatment with PF-05280014, a potential trastuzumab biosimilar, was non-inferior to trastuzumab-EU, based on pharmacokinetic data. This objective was met; the study demonstrated non-inferiority for PF-05280014 versus trastuzumab-EU in the percentage of patients with Cycle $5 C_{\text {trough }}$ (Cycle 6 predose) $>20 \mu \mathrm{g} / \mathrm{ml}$. Furthermore, results from an analysis of secondary endpoints demonstrated comparable pharmacokinetic profiles for PF-05280014 and trastuzumab-EU.

PF-05280014 and trastuzumab-EU also had comparable efficacy profiles, based on measures of tumour control, with no notable difference between treatment groups (PF-05280014 vs trastuzumab-EU, respectively) in pCR rate $(47.0 \%$ vs $50.0 \%)$ or ORR $(88.1 \%$ vs $82.0 \%)$. Importantly, $\mathrm{pCR}$ results were consistent with published data for trastuzumab administered in combination with neoadjuvant chemotherapy. ${ }^{27,28}$ Furthermore, both $\mathrm{PCR}$ and ORR were comparable to rates reported for other proposed trastuzumab biosimilars in development and evaluated in the neoadjuvant setting. ${ }^{29,30}$

Both treatments were generally well tolerated, with few patients discontinuing from any treatment due to AEs. There was no clinically significant imbalance between groups in TEAEs, serious TEAEs or other observed safety parameters. PF-05280014 and trastuzumab-EU also had comparable immunogenicity profiles, wherein no patients in the PF-05280014 group and one patient in the trastuzumab-EU group tested positive for ADAs. These data are consistent with previous studies demonstrating a low immunogenic potential for trastuzumab. ${ }^{19,31}$ As part of a stepwise comparison exercise to demonstrate biosimilarity, these findings build on those of previous analytical and non-clinical studies and two single-dose studies in healthy volunteers. ${ }^{18-20}$

The current study applied a non-inferiority design to compare PF-05280014 with trastuzumab-EU. Generally, biosimilarity studies use an equivalence design to show the biosimilar is neither superior nor inferior to the originator product and vice versa. ${ }^{16,17,32}$ However, in some cases, a non-inferiority design may be adequate to demonstrate that there are no clinically meaningful differences between the proposed biosimilar product and originator biologic. ${ }^{16}$ This study may be limited by its use of a margin for non-inferiority of the clinical endpoint $\mathrm{PCR}$ to establish non-inferiority using a primary endpoint based on pharmacokinetics data. However, a non-inferiority margin based on clinical considerations rather than statistical considerations is supported 
Table 3. Pathological response and overall tumour response assessments (per protocol population)

\begin{tabular}{|c|c|c|}
\hline & $\begin{array}{l}\text { PF-05280014 } \\
(n=101)\end{array}$ & $\begin{array}{l}\text { Trastuzumab-EU } \\
(n=89)\end{array}$ \\
\hline \multicolumn{3}{|c|}{ Pathological response assessment } \\
\hline \multicolumn{3}{|l|}{ Response category, $n$ (\%) } \\
\hline $\mathrm{pCR}$ & $47(46.5)$ & $43(48.3)$ \\
\hline pPR & $51(50.5)$ & $40(44.9)$ \\
\hline No pathological response & $2(2.0)$ & $3(3.4)$ \\
\hline Not done $\mathrm{a}^{\mathrm{a}}$ & $1(1.0)$ & $3(3.4)$ \\
\hline \multicolumn{3}{|l|}{ Patients who had surgery } \\
\hline$n(\%)$ & $100(99.0)$ & 86 (96.6) \\
\hline Patients with $\mathrm{pCR}, \mathrm{b} n(\%)$ & $47(47.0)$ & $43(50.0)$ \\
\hline $95 \% \mathrm{Cl}$ & $36.9,57.2$ & $39.0,61.0$ \\
\hline $\begin{array}{l}\text { Stratified difference in } \mathrm{pCR} \\
\text { between PF-05280014 and } \\
\text { trastuzumab-EUc }\end{array}$ & \multicolumn{2}{|c|}{-2.81} \\
\hline $\begin{array}{l}\text { Standard error for the } \\
\text { difference }\end{array}$ & \multicolumn{2}{|c|}{7.03} \\
\hline $\begin{array}{l}95 \% \mathrm{Cl} \text { (stratified) for the } \\
\text { difference }\end{array}$ & \multicolumn{2}{|c|}{$-16.58,10.96$} \\
\hline \multicolumn{3}{|c|}{ Overall response assessment (per central radiology review) } \\
\hline \multicolumn{3}{|c|}{ Overall response category at Cycle 6/EOT, $n(\%)$} \\
\hline Complete response & $3(3.0)$ & 0 \\
\hline Partial response & $86(85.1)$ & $73(82.0)$ \\
\hline Stable disease & $7(6.9)$ & $4(4.5)$ \\
\hline Progressive disease & $2(2.0)$ & $1(1.1)$ \\
\hline Non-evaluable & $1(1.0)$ & $6(6.7)$ \\
\hline $\begin{array}{l}\text { Non-complete response/ } \\
\text { non-progressive disease }\end{array}$ & $1(1.0)$ & $3(3.4)$ \\
\hline Missing & $1(1.0)$ & $2(2.2)$ \\
\hline \multicolumn{3}{|l|}{ ORR $^{d}$} \\
\hline$n(\%)$ & $89(88.1)$ & $73(82.0)$ \\
\hline $95 \% \mathrm{Cl}$ & $80.2,93.7$ & $72.5,89.4$ \\
\hline $\begin{array}{l}\text { Stratified difference in ORR } \\
\text { between PF-05280014 and } \\
\text { trastuzumab-EUc }\end{array}$ & \multicolumn{2}{|c|}{5.96} \\
\hline $\begin{array}{l}\text { Standard error for the } \\
\text { difference }\end{array}$ & \multicolumn{2}{|c|}{5.09} \\
\hline $\begin{array}{l}95 \% \mathrm{Cl} \text { (stratified) for the } \\
\text { difference }\end{array}$ & \multicolumn{2}{|c|}{$-4.01,15.94$} \\
\hline
\end{tabular}

aPathology data were not recorded or response was not assessed for the following reasons: completed the study but had no surgery (PF-05280014, $n=1$; trastuzumab-EU, $n=1$ ) or completed treatment but lost to follow-up prior to surgery (trastuzumab-EU, $n=2$ ). ${ }^{\text {b } T h e ~ d e n o m i n a t o r s ~ f o r ~ p e r c e n-~}$ tages of patients with $\mathrm{pCR}$ included only patients who had surgery. 'Stratified analysis was based on the normal approximation to the binomial distribution, adjusting for randomisation strata (primary tumour size $<5 \mathrm{~cm}$ vs $\geq 5 \mathrm{~cm}$; oestrogen receptor-positive vs oestrogen receptornegative; and progesterone receptor-positive vs progesterone receptornegative). ${ }^{\mathrm{d}} \mathrm{ORR}$ was defined as the percentage of patients within each treatment group who achieved complete response or partial response by Cycle 6/EOT, in accordance with Response Evaluation Criteria in Solid Tumours, version 1.1. $\mathrm{Cl}=$ confidence interval; $\mathrm{EOT}=$ end of treatment; $\mathrm{ORR}=$ objective response rate; $\mathrm{pCR}=$ pathological complete response; $\mathrm{pPR}=$ pathological partial response; trastuzumab-EU = licensed trastuzumab sourced from the European Union

by a lack of previous evidence in the neoadjuvant setting outside of the HannaH trial. ${ }^{26}$ In addition, this study was not powered to evaluate non-inferiority in $\mathrm{PCR}$ or ORR, which may limit interpretation of clinical efficacy findings. However, a comparative
Table 4. All-causality, treatment-emergent adverse events (safety population) ${ }^{\mathrm{a}}$

\begin{tabular}{|c|c|c|}
\hline & $\begin{array}{l}\text { PF-05280014 } \\
(n=113)\end{array}$ & $\begin{array}{l}\text { Trastuzumab-EU } \\
(n=112)\end{array}$ \\
\hline Number of AEs & 569 & 511 \\
\hline \multicolumn{3}{|l|}{ Patients with event, $n$ (\%) } \\
\hline AEs & $109(96.5)$ & $106(94.6)$ \\
\hline SAEs ${ }^{b}$ & $7(6.2)$ & $6(5.4)$ \\
\hline Grade 3 or $4 \mathrm{AEs}$ & $43(38.1)$ & $51(45.5)$ \\
\hline Grade 5 AEs & $1(0.9)$ & 0 \\
\hline Discontinued study due to AEs & $1(0.9)$ & $3(2.7)$ \\
\hline $\begin{array}{l}\text { Discontinued from any } \\
\text { treatment }^{c} \text { due to AEs }\end{array}$ & $4(3.5)$ & $3(2.7)$ \\
\hline $\begin{array}{l}\text { Dose reduced or temporarily } \\
\text { discontinued for any } \\
\text { treatment }^{c} \text { due to AEs }\end{array}$ & $37(32.7)$ & $30(26.8)$ \\
\hline \multicolumn{3}{|c|}{ 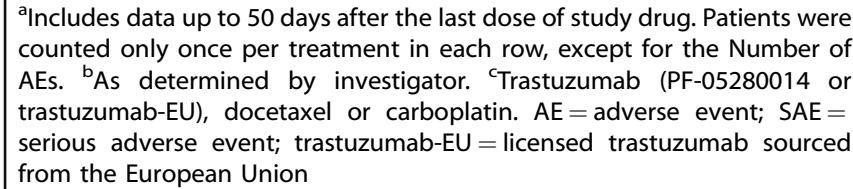 } \\
\hline
\end{tabular}

Table 5. Incidence of ADAs by visit and overall (safety population) ${ }^{\mathrm{a}}$

\begin{tabular}{llll}
\hline Visit & ADA status & PF-05280014 & Trastuzumab-EU \\
\hline Cycle 1 & Patients assessed, $n$ & 113 & 112 \\
& Negative $<1.00, n(\%)$ & $113(100.00)$ & $110(98.21)$ \\
& Positive $\geq 1.00, n(\%)$ & 0 & $1(0.89)$ \\
& Not analysed, $n(\%)$ & 0 & $1(0.89)$ \\
Cycle 2 & Patients assessed, $n$ & 111 & 112 \\
& Negative $<1.00, n(\%)$ & $111(100.00)$ & $112(100.00)$ \\
& Positive $\geq 1.00, n(\%)$ & 0 & 0 \\
Cycle 4 & Patients assessed, $n$ & 108 & 109 \\
& Negative $<1.00, n(\%)$ & $108(100.00)$ & $109(100.00)$ \\
& Positive $\geq 1.00, n(\%)$ & 0 & 0 \\
Cycle 6 & Patients assessed, $n$ & 108 & 108 \\
& Negative $<1.00, n(\%)$ & $108(100.00)$ & $108(100.00)$ \\
& Positive $\geq 1.00, n(\%)$ & 0 & 0 \\
Overall & Patients assessed, $n$ & 113 & 112 \\
& Negative $<1.00, n(\%)$ & $113(100.00)$ & $111(99.11)$ \\
& Positive $\geq 1.00, n(\%)$ & 0 & $1(0.89)$
\end{tabular}

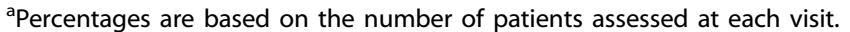
The unit of ADA titre was endpoint titre. Only predose assessments are summarised. $A D A=$ antidrug antibody; trastuzumab-EU = licensed trastuzumab sourced from the European Union

study of PF-05280014 versus trastuzumab-EU, each in combination with paclitaxel, as first-line treatment in patients with HER2+ metastatic breast cancer used an equivalence design, and preliminary results demonstrated that this trial met its primary endpoint of similarity in ORR. ${ }^{33}$

The strengths of the current trial include its randomised, double-blind design, homogeneous patient population and use of clinical endpoints that measure activity (e.g., tumour response). The most frequently published definition of $\mathrm{pCR}$ (absence of invasive neoplastic cells in breast and lymph nodes following 
neoadjuvant therapy) was used, consistent with previous studies of trastuzumab administered concurrently with chemotherapy in the neoadjuvant setting. ${ }^{11,12,26}$ Furthermore, calculation of ORR for efficacy analysis was based on independent central radiologic review of tumour assessments. Finally, neoadjuvant use of trastuzumab is not currently approved by the US Food and Drug Administration"; however, it is authorised by the European Medicines Agency and is well known and used in clinical practice in Europe and the United States. 2,25,34,35 Therefore, the finding of comparable efficacy in terms of $\mathrm{pCR}$ and ORR in this setting may be reassuring for patients and clinicians.

In conclusion, neoadjuvant treatment with the potential biosimilar PF-05280014 administered in combination with docetaxel and carboplatin demonstrated non-inferiority in pharmacokinetics and comparability in efficacy, safety and immunogenicity when compared with trastuzumab-EU in combination with docetaxel and carboplatin in patients with operable HER2+ breast cancer. These results support similarity of PF-05280014 to trastuzumab-EU as part of the stepwise comparison exercise for demonstrating biosimilarity. As a potential biosimilar of trastuzumab, PF-05280014 could broaden the number of treatment options for patients with HER2+ breast cancer and allow greater use of anti-HER2 therapy across clinical settings.

\section{ACKNOWLEDGEMENTS}

Medical writing support was provided by Elyse Smith, PhD, of Engage Scientific Solutions and funded by Pfizer Inc. This study was funded by Pfizer Inc. Trial registration: ClinicalTrials.gov: NCT02187744; EU Clinical Trials Register EudraCT number: 2013-004679-11.

\section{AUTHOR CONTRIBUTIONS}

Richat Abbas, Fiona Hilton, Jennifer Coppola and Ira Jacobs contributed to study design and conduct. Fiona Hilton analysed the data and provided statistical support. Philip E. Lammers, Magdolna Dank and Riccardo Masetti contributed to acquisition of data. All authors made substantial contributions to interpretation of data, were involved in drafting the manuscript and/or revising it critically for important intellectual content, approved the final version for submission and agree to be accountable for all aspects of the work.

\section{ADDITIONAL INFORMATION}

Supplementary information is available for this paper at https://doi.org/10.1038/ s41416-018-0147-1.

Ethics approval and consent to participate: The study was conducted in compliance with the provisions of the Declaration of Helsinki and in accordance with international standards of Good Clinical Practice. All patients provided informed consent prior to undergoing screening procedures. The final protocol, amendments and informed consent documentation were reviewed and approved by an institutional review board or independent ethics committee(s) at each of the participating investigational sites.

Funding: This study was funded by Pfizer Inc.

Competing interests: Philip E. Lammers has participated on advisory boards with Pfizer Inc. Magdolna Dank has been a member of Biosimilars Oncology European Advisory Board with Pfizer Inc since 2013. Riccardo Masetti declares no conflicts of interest. Richat Abbas, Fiona Hilton, Jennifer Coppola and Ira Jacobs are full time employees of and declare stock holdings and/or stock options from Pfizer Inc.

Availability of data and materials: Pfizer's policies on the provision of clinical trial data are set out on our website: http://www.pfizer.com/research/clinical trials/ trial_data_and_results. In addition to posting clinical trial results on the ClinicalTrials. gov registry, Pfizer will provide access to anonymised patient-level data in response to scientifically valid research protocols. Data from Pfizer-sponsored global interventional clinical studies are available from: trials conducted for medicines, vaccines and medical devices for indications that have been approved in the US and/ or EU; trials conducted for medicines, vaccines and medical devices that have been terminated (i.e., development for all indications has been discontinued). Data from these trials will be made available 24 months after study completion. Pfizer will make reasonable efforts to fulfil all data requests for legitimate research purposes, but there may be instances in which retrieval or delivery of data is not feasible (for example, if Pfizer does not have legal authority to provide the data, if costs of retrieval of older or pre-electronic data are prohibitive, etc. See page $5^{*}$ at the following link: https://www.pfizer.com/files/research/research_clinical_trials/ A_Guide_to_Requesting_Pfizer_Patient-Level_Clinical_Trial_Data_2017.pdf). Further detail can be found at: http://www.pfizer.com/research/clinical_trials/ trial_data_and_results/data_requests. Pfizer's practices adhere to the principles for responsible data sharing laid out by the European Federation of Pharmaceutical Industries and Associations (EFPIA) and the Pharmaceutical Research and Manufacturers of America (PhRMA): http://phrma.org/sites/default/files/pdf/ PhRMAPrinciplesForResponsibleClinicalTrialDataSharing.pdf.

Publisher's note: Springer Nature remains neutral with regard to jurisdictional claims in published maps and institutional affiliations.

\section{REFERENCES}

1. Genentech Inc. Herceptin (trastuzumab) US prescribing information. http://www. gene.com/download/pdf/herceptin_prescribing.pdf. Accessed 11 July 2017 (2017).

2. European Medicines Agency. Herceptin (trastuzumab) summary of product characteristics. http://www.ema.europa.eu/ema/index.jsp?curl=pages/medicines/ human/medicines/000278/human_med_000818.jsp\&mid=WC0b01 ac058001d124. Accessed 13 July 2017 (2013).

3. Press, M. F. et al. Her-2/neu expression in node-negative breast cancer: direct tissue quantitation by computerized image analysis and association of overexpression with increased risk of recurrent disease. Cancer Res. 53, 4960-4970 (1993).

4. Seshadri, R. et al. Clinical significance of HER-2/neu oncogene amplification in primary breast cancer. The South Australian Breast Cancer Study Group. J. Clin. Oncol. 11, 1936-1942 (1993).

5. Slamon, D. J. et al. Human breast cancer: correlation of relapse and survival with amplification of the HER-2/neu oncogene. Science 235, 177-182 (1987).

6. Wolff, A. C. et al. Recommendations for human epidermal growth factor receptor 2 testing in breast cancer: American Society of Clinical Oncology/College of American Pathologists clinical practice guideline update. J. Clin. Oncol. 31, 3997-4013 (2013)

7. Piccart-Gebhart, M. J. et al. Trastuzumab after adjuvant chemotherapy in HER2positive breast cancer. N. Engl. J. Med. 353, 1659-1672 (2005).

8. Romond, E. H. et al. Trastuzumab plus adjuvant chemotherapy for operable HER2positive breast cancer. N. Engl. J. Med. 353, 1673-1684 (2005).

9. Slamon, D. et al. Adjuvant trastuzumab in HER2-positive breast cancer. N. Engl. J. Med. 365, 1273-1283 (2011).

10. Slamon, D. J. et al. Use of chemotherapy plus a monoclonal antibody against HER2 for metastatic breast cancer that overexpresses HER2. N. Engl. J. Med. 344, 783-792 (2001).

11. Buzdar, A. U. et al. Significantly higher pathologic complete remission rate after neoadjuvant therapy with trastuzumab, paclitaxel, and epirubicin chemotherapy: results of a randomized trial in human epidermal growth factor receptor 2positive operable breast cancer. J. Clin. Oncol. 23, 3676-3685 (2005).

12. Gianni, L. et al. Neoadjuvant chemotherapy with trastuzumab followed by adjuvant trastuzumab versus neoadjuvant chemotherapy alone, in patients with HER2-positive locally advanced breast cancer (the NOAH trial): a randomised controlled superiority trial with a parallel HER2-negative cohort. Lancet 375, 377-384 (2010).

13. Cherny, N., Sullivan, R., Torode, J., Saar, M. \& Eniu, A. ESMO European Consortium Study on the availability, out-of-pocket costs and accessibility of antineoplastic medicines in Europe. Ann. Oncol. 27, 1423-1443 (2016).

14. Lammers, P., Criscitiello, C., Curigliano, G. \& Jacobs, I. Barriers to the use of trastuzumab for HER2 + breast cancer and the potential impact of biosimilars: a physician survey in the United States and emerging markets. Pharmaceuticals (Basel) 7, 943-953 (2014).

15. European Medicines Agency. Guideline on similar biological medicinal products. http://www.ema.europa.eu/docs/en_GB/document_library/Scientific_guideline/ 2014/10/WC500176768.pdf. Accessed 11 July 2017 (2014).

16. US Food and Drug Administration. Scientific considerations in demonstrating biosimilarity to a reference product. Guidance for industry. http://www.fda.gov/ downloads/Drugs/GuidanceComplianceRegulatoryInformation/Guidances/ UCM291128.pdf. Accessed 11 July 2017 (2015).

17. World Health Organization. Guidelines on evaluation of similar biotherapeutic products (SBPs). http://www.who.int/biologicals/areas/biological_therapeutics/ BIOTHERAPEUTICS FOR WEB 22APRIL2010.pdf. Accessed 11 July 2017 (2009). 
18. Hurst, S. et al. Comparative nonclinical assessments of the proposed biosimilar PF-05280014 and trastuzumab (Herceptin((R))). BioDrugs 28, 451-459 (2014).

19. Yin, D. et al. A randomized phase 1 pharmacokinetic trial comparing the potential biosimilar PF-05280014 with trastuzumab in healthy volunteers (REFLECTIONS B327-01). Br. J. Clin. Pharmacol. 78, 1281-1290 (2014).

20. Vana, A. M. et al. Evaluating imbalances of adverse events during biosimilar development. Mabs 8, 861-866 (2016).

21. ClinicalTrials.gov. A study of PF-05280014 [trastuzumab-Pfizer] or Herceptin ${ }^{\circledR}$ [trastuzumab-EU] plus paclitaxel in HER2 positive first line metastatic breast cancer treatment (REFLECTIONS B327-02). https://clinicaltrials.gov/ct2/show/ NCT01989676. Accessed 22 March 2017 (2013).

22. ClinicalTrials.gov. A study of PF-05280014 or trastuzumab plus Taxotere ${ }^{\circledR}$ and carboplatin in HER2 positive breast cancer in the neoadjuvant setting (REFLECTIONS B327-04). https://clinicaltrials.gov/ct2/show/NCT02187744?term $=P F$ $+05280014 \&$ rank $=4$. Accessed 11 July 2017 (2014).

23. EU Clinical Trials Register. A randomized, double-blind pharmacokinetic study of PF-05280014 plus Taxotere and carboplatin versus Herceptin plus Taxotere and carboplatin for the neoadjuvant treatment of patients with operable HER2positve breast cancer. https://www.clinicaltrialsregister.eu/ctr-search/search? query=2013-004679-11. Accessed 11 July 2017 (2014)

24. Coudert, B. P. et al. Multicenter phase II trial of neoadjuvant therapy with trastuzumab, docetaxel, and carboplatin for human epidermal growth factor receptor-2-overexpressing stage II or III breast cancer: results of the GETN(A)-1 trial. J. Clin. Oncol. 25, 2678-2684 (2007).

25. National Comprehensive Cancer Network. NCCN Clinical Practice Guidelines in Oncology: breast cancer, version 3.2013. https://www.nccn.org/. Accessed 11 July 2017 (2015).

26. Ismael, G. et al. Subcutaneous versus intravenous administration of (neo)adjuvant trastuzumab in patients with HER2-positive, clinical stage I-III breast cancer (HannaH study): a phase 3, open-label, multicentre, randomised trial. Lancet Oncol. 13, 869-878 (2012).

27. Untch, M. et al. Lapatinib versus trastuzumab in combination with neoadjuvant anthracycline-taxane-based chemotherapy (GeparQuinto, GBG 44): a randomised phase 3 trial. Lancet Oncol. 13, 135-144 (2012).

28. Untch, M. et al. Neoadjuvant treatment with trastuzumab in HER2-positive breast cancer: results from the GeparQuattro study. J. Clin. Oncol. 28, 2024-2031 (2010).

29. Stebbing, J. et al. CT-P6 compared with reference trastuzumab for HER2-positive breast cancer: a randomised, double-blind, active-controlled, phase 3 equivalence trial. Lancet Oncol. 8, 917-928 (2017).
30. Pivot, X. B. et al. A randomized, double-blind, phase III study comparing SB3 (trastuzumab biosimilar) with originator trastuzumab in patients treated by neoadjuvant therapy for HER2-positive early breast cancer [abstract]. J. Clin. Oncol. 35, 509 (2017).

31. Wynne, C. et al. Comparison of subcutaneous and intravenous administration of trastuzumab: a phase $\mathrm{I} / \mathrm{lb}$ trial in healthy male volunteers and patients with HER2positive breast cancer. J. Clin. Pharmacol. 53, 192-201 (2013).

32. Isakov, L., Jin, B. \& Jacobs, I. A. Statistical primer on biosimilar clinical development. Am. J. Ther. 23, e1903-e1910 (2016).

33. Pegram M., et al. (2017) A randomized, double-blind study of PF-05280014 (a potential trastuzumab biosimilar) vs trastuzumab, both in combination with paclitaxel, as first-line treatment for HER2-positive metastatic breast cancer. Presented at: European Society for Medical Oncology (ESMO 2017), Madrid, Spain, 08-12 September 2017.

34. Denduluri, N. et al. Selection of optimal adjuvant chemotherapy regimens for early breast cancer and adjuvant targeted therapy for human epidermal growth factor receptor 2-positive breast cancers: an American Society of Clinical Oncology Guideline adaptation of the Cancer Care Ontario Clinical Practice Guideline. J. Clin. Oncol. 34, 1-12 (2016).

35. Senkus, E. et al. Primary breast cancer: ESMO Clinical Practice Guidelines for diagnosis, treatment and follow-up. Ann. Oncol. 26, v8-v30 (2015).

Open Access This article is licensed under a Creative Commons cc) Attribution 4.0 International License, which permits use, sharing, adaptation, distribution and reproduction in any medium or format, as long as you give appropriate credit to the original author(s) and the source, provide a link to the Creative Commons license, and indicate if changes were made. The images or other third party material in this article are included in the article's Creative Commons license, unless indicated otherwise in a credit line to the material. If material is not included in the article's Creative Commons license and your intended use is not permitted by statutory regulation or exceeds the permitted use, you will need to obtain permission directly from the copyright holder. To view a copy of this license, visit http://creativecommons. org/licenses/by/4.0/.

(c) The Author(s) 2018 